\title{
Ein Verfahren zur Beurteilung konservierungs- und sterilisationsbedingter Kollagenschädigungen an homologen und heterologen Herzklappen- und Duratransplantaten
}

\author{
Von H. HAas, F. W. HehrLein und L. RóKA ${ }^{1}$ ) \\ Aus dem Institut für Klinische Chemie (Direktor: Professor Dr. L. Róka) und der Chirurgischen Universitätsklinik der \\ Justus-Liebig-Universität Gießen (Direktor: Professor Dr. K. Voßschulte)
}

(Eingegangen am 29. November 1969)

\begin{abstract}
Es wird über ein Verfahren berichtet, welches eine differenzierte Beurteilung konservierungsbedingter Kollagenschädigung an Aortenklappentransplantaten und Duratransplantaten ermöglicht. Durch Bestimmung des durch Trypsin-Andauung freigesetzten Hydroxyprolin der untersuchten Gewebe lassen sich deutlich unterschiedliche Transplantatschädigungen nachweisen.
\end{abstract}

A metbod for the determination of collagen damage caused by preservation and sterilisation in bomologous and beterologous transplants of heart valves and dura

A method is reported for the differential assessment of collagen damage caused by preservation in aorta valve transplants and dura transplants. Markedly different degrees of transplant damage are revealed by measuring the hydroxyproline released by trypsin digestion of the tissue.

Die Verwendung autologen Gewebes in der Transplantationschirurgie ist mit dem Nachteil eines verlängerten operativen Eingriffes verbunden und nur dann möglich, wenn das entnommene Transplantat am Entnahmeort keine wesentliche funktionelle Störung hinterläßt. Kunststoffmaterialien genügen oft nicht den an sie gestellten Anforderungen bezüglich der Belastbarkeit auf lange Zeit und der Gewebeverträglichkeit. Aus diesem Grunde gewinnt die Anwendung homologer und heterologer Transplantate in verschiedenen Disziplinen der Chirurgie in den letzten Jahren eine immer größere Bedeutung. So hat sich vor allem im kardio-vaskulären Bereich der Ersatz der erkrankten Aorten- und Mitralklappe durch menschliche und tierische Aortenklappentransplantate bewährt, und bildet heute einen Gegenpol zu dem sonst üblichen Herzklappenersatz durch künstliche Kugel- oder Linsen-Ventilprothesen (1-7).

In der Neurochirurgie stellt die Anwendung von vorgefertigter Fremddura zur Deckung großer Duradefekte eine wesentliche Bereicherung der operativen Behandlungsmöglichkeiten dar (8-10).

Diese homologen und heterologen Transplantate sind im wesentlichen zellfreies biologisches Material. Sie werden ohne Berücksichtigung einer Antigenität übertragen.

$\mathrm{Um}$ sie in ausreichender Menge für die notwendigen Eingriffe bereitstellen zu können, mußten Konservierungsverfahren entwickelt werden, die eine längerdauernde Aufbewahrung der Transplantate in einer sog. "Gewebebank" ermöglichen. Zusätzliche Sterilisationsmaßnahmen sind bei der meist unter unsterilen Bedingungen : tattfindenden Transplantatgewinnung exforderlich.

\footnotetext{
1) Techn. Assistenz Karen Drohmann.
}

Die Gefriertrocknung gilt seit ihrer Einführung in den klinischen Bereich durch FLosDorf und MudD (11) im Jahre 1935 als ein zuverlässiges Verfahren der Langzeitkonservierung für biologisches Gewebe. Unter den zur V.erfügung stehenden Sterilisaționsmitteln hat sich vor allem die Äthylenoxidbegasung (12) bewährt. Verbreitete Anwendung fanden ferner die Strahlensterilisation und $\beta$-Propiolacton-Behandlung $(13,14)$. Zur sterilen Feuchtkonservierung sind vor allem Lösungen von Quecksilberverbindungen geeignet (5).

Alle aufgeführten Konservierungs- und Sterilisationsverfahren rufen jedoch morphologisch faßbare, mehr oder minder starke Veränderungen am Gewebe hervor (15). Von besonderem klinischen Interesse erschien uns deshalb die Beurteilung einer evtl. konservierungsbedingten Wertminderung bei Herzklappen- und Duratransplantaten, denn Aortenklappen und Duren sind, wie bereits erwähnt, zur Transplantation besonders geeignet.

Im folgenden wird ein Verfahren beschrieben, das eine differenzierte Aussage über den Grad der Kollagenschädigung an diesen Geweben aufgrund der vorangegangenen Konservierungsprozesse ermöglicht. Wir gehen dabei davon aus, daß natives Kollagen durch Verdauungs-Proteasen kaum gespalten wird (16). Denaturiertes Kollagen verliert aber sein ursprüngliches molekulares Gefüge und wird für Trypsin leicht angreifbar. Wenn man voraussetzt, daß alle Konservierungs- und Sterilisationsverfahren mit einer unterschiedlich starken Denaturierung des biologischen Materials einhergehen, so muß aus der quantitativ unterschiedlichen Angreifbarkeit für Trypsin das Ausmaß der Schädigung durch die einzelnen Verfahren ablesbar sein. 


\section{Methodik}

\section{Material}

Kälberaorten, menschliche Dura.

L(-)-Hydroxyprolin (Merck 4506)

Chloramin T (Merck 2426)

4-Dimethylaminobenzaldehyd (Merck 3057)

Perchlorsäure, 4M (durch Verdünnen aus $\mathrm{HClO}_{4} 70$ proz., Merck 519)

Trypsin (Serva 37260)

\section{Lösungen}

Citrat-Acetat-Puffer $p H 6$

$50 \mathrm{~g}$ Citronensäure $\cdot \mathrm{H}_{2} \mathrm{O}$ (Merck 244)

$120 \mathrm{~g} \mathrm{Na}$-Acetat $3 \mathrm{H}_{2} \mathrm{O}$ (Merck 6495)

$34 \mathrm{~g} \mathrm{NaOH}$

$12 \mathrm{~m} l$ Eisessig ad $1000 \mathrm{~m} l$ redest. Wasser

Chloramin T-Lösung

$2,82 \mathrm{~g}$ Chloramin $\mathrm{T}$

$40 \mathrm{ml}$ redest. Wasser

$60 \mathrm{ml}$ Äthylenglycolmonomethyläther (Merck 858)

$100 \mathrm{~m} l$ Citrat-Acetat-Puffer

\section{p-Dimetbylaminobenzaldebyd}

$10 \mathrm{~g}$ in $100 \mathrm{ml}$ A thylenglycolmonomethyläther lösen

1 proz. $\beta$-Propiolacton Lösung in Wasser

\section{Cialitlösung nach CARPENTIER}

Stammlösung:

24,2 $\mathrm{g}$ Trishydroxymethylaminomethan

$23,2 \mathrm{~g}$ Maleinsäure aufgefüllt auf $1000 \mathrm{ml}$ redest. Wasser, welches vorhex zur Entfernung des $\mathrm{CO}_{2}$ zum Sieden gebracht worden ist.

Eigentliche Konservierungslösung:

$250 \mathrm{ml}$ Stammlösung

$240 \mathrm{ml}$ 0,2N Natriumbicarbonat

$0,01 \mathrm{~g}$ 2-Äthylenmercurimercaptobenzoxazol-5-carbonsaures $\mathrm{Na}$ trium

$0,05 \mathrm{~g}$ Phenolrot

ad $1000 \mathrm{~m} l$ redest. Wasser

\section{Trypsin-Puffer $p H 8$}

$\mathrm{Zu} 100 \mathrm{ml}$ 1N Ameisensäure wird 1N Ammoniaklösung unter Rühren, bis $\mathrm{pH} 8$ erreicht ist, zugesetzt. In $50 \mathrm{~m} /$ Puffer werden jeweils frisch $100 \mathrm{mg}$ Trypsin gelöst.

\section{Antibiotika-Heparin-Lösung}

$1 \mathrm{~g}$ Streptomycin und $10000 \mathrm{E}$ Heparin in $1 /$ physiol. $\mathrm{NaCl}$ Lösung.

\section{Vorgehen}

Je 20 Kälberaortenklappen wurden bis spätestens 6 Stdn. nach der Schlachtung dex Tiere vom anhängenden Gewebe freipräpariert und zunächst für $12 \mathrm{Stdn}$. in einer $\mathrm{NaCl}$-Streptomycin-HeparinLösung aufbewahrt, dann entweder nur lyophilisiert, oder einerseits vor der Gefriertrocknung $12 \mathrm{Stdn}$. mit Äthylenoxid begast oder $1 \mathrm{Std}$. in eine 1proz. $\beta$-Propiolacton-Lösung eingelegt oder andererseits nach der Gefriertrocknung mit einer Gesamtdosis von 2,5 Mrad gammastrahlensterilisiert.

Ein weiteres Segment haben wir für 4 Wochen in eine äquilibrierte Cialitlösung nach der Methode Carpenter-Audhour eingelegt.

Die so vorbehandelten Klappensegmente wurden gewogen, mit der Schere zerkleinert und unter leichtem Rühren mit $10 \mathrm{mg}$ Trypsin in $5 \mathrm{ml}$ Puffer pro Klappensegment $12 \mathrm{Stdn}$. bei $37^{\circ}$ auf einem Magnetrührer zur Verdauung angesetzt.

Nach Abpipettieren des Uberstandes und zweimaligem Waschen des Rückstandes wurde das Waschwasser zusammen mit dem Uberstand auf $10 \mathrm{~m} l$ aufgefüllt. Getrennt wurden dann $2 \mathrm{~m} l$ des Uberstandes und der gesamte Rückstand mit je $2 \mathrm{ml} 25 \mathrm{proz}$. Salzsäure in Ampullen eingeschmolzen und $20 \mathrm{Stdn}$. bei $110^{\circ} \mathrm{im}$ Brutschrank hydrolysiert.
Nach der Hydrolyse wurden die Ansätze mit Citrat-Acetat-Puffer versetzt und mit dem Titrator ${ }^{2}$ ) auf $\mathrm{pH} 6$ cingestellt. Die mit Cialit behandelten Proben mußten zur Beseitigung des Phenolrotindikators über eine kurze Amberlite Säule (Amberlite IRA 410, Serva, in Transfusionsschlauch, Durchmesser $3 \mathrm{~mm}, 100 \mathrm{~mm}$ Höhe) gegeben werden.

Der Hydroxyprolingehalt wurde nach der Methode STrgemanN bestimmt, die Extinktion des dabei entstehenden roten Farbstoffs mit dem Filter $\mathrm{Hg} 578 \mathrm{~nm}$ am Eppendorf-Fotometer ${ }^{3}$ ) abgelesen.

\section{Auswertung}

Mit $20 \mathrm{mg} \mathrm{L}(-)$-Hydroxyprolin in $100 \mathrm{ml} 0,001 \mathrm{~N} \mathrm{HCl}$ als Stammlösung wurde eine Eichkurve erstellt, die Extinktionen der Proben in $\mathrm{mg}$ abgelesen, entsprechend den Verdünnungen mulpliziert und auf $1 \mathrm{~g}$ Klappeneinwage berechnet. Das Maß der Schädigung errechnet sich aus dem prozentualen Anteil des Hydroxyprolins im Uberstand vom Gesamthydroxyprolin.

Tab. 1

Hydroxyprolingehalt des Öberstandes nach Trypsinierung, ausgedruckt in Prozent des Gesamthydroxyprolin des Klappensegmentes. Angegeben sind Mittelwerte und Streuung der sechs verschiedenen Gruppen sowie die Irrtumswahrscheinlichkeit

\begin{tabular}{lccrc}
\hline & $\mathrm{n}$ & $\overline{\mathbf{x}}$ & \multicolumn{1}{c}{$\pm \mathrm{s}$} & $\mathrm{p}$ \\
\hline Nativ & 20 & 38,73 & 6,45 & - \\
Lyophilisiert & 20 & 42,54 & 10,02 & n.s. \\
$\beta$-Propiolacton und lyophilisiert & 20 & 65,62 & 10,54 & 0,001 \\
Lyophilisiert und 2,5 M rad & 20 & 63,27 & 13,95 & 0,001 \\
\&thylenoxid und lyophilisiert & 20 & 17,20 & 7,45 & 0,001 \\
Cialitkonservierung & 20 & 10,60 & 5,54 & 0,001 \\
\hline
\end{tabular}

Die Unabhängigkeit der Mittelwerte der einzelnen behandelten Gruppen haben wir mit der Einweg-VarianzAnalyse bestätigt:

Tab. 2

Einweg-Varianz-Analyse. Angegeben sind die Summe der Quadrate (SS) zwischen und innerhalb der Gruppen, die Zahl der Freiheitsgrade (di), die mittlere Summe der Quadrate (MS $\left.=\mathrm{SS}_{\text {f }}\right)$ und F $\left(=\frac{M S \mathrm{zW}}{M S \text { in }}\right)$. *** Die Signifikanz ist sehr hoch

\begin{tabular}{lcrrc}
\hline Quelle & SS & df & MS & F \\
\hline zw.*** & 51768,169 & 4 & 12942,042 & $131,448 * * *$ \\
inner. & 9353,650 & 95 & 98,457 & - \\
Total & 61121,630 & 99 & & - \\
\hline
\end{tabular}

Die statistische Sicherung der Befunde der behandelten Klappensegmente gegenüber dem nativen Präparat erfolgte mit dem $t$-Test nach Student. Die partielle Abhängigkeit der Einzelgruppen, die durch die gleiche Bezugsgruppe gegeben ist, dürfte bei der sehr starken Signifikanz nicht ins Gewicht fallen.

\section{Diskussion}

Kollagenes Fasergewebe ist durch seinen Gehalt an Hydroxyprolin gewissermaßen biologisch markiert. Hydroxyprolin kommt im Organismus nur noch im Elastin in geringen Mengen vor.

Eine spezifische und telativ empfindliche Nachweismethode für Hydroxyprolin besteht in der Umwandlung der Aminosäure in Pyrrol durch Decarboxylierung und Wasserabspaltung, mit nachfolgender Reaktion des Pyrrols mit $p$-Dimethylaminobenzaldehyd, wobei ein roter Farbstoff gebildet wird.

2) Titrator und Autoburette Radiometer Copenhagen.

3) Fa. Netheler und Hinz, Hamburg. 
Von verschiedenen Autoren wurden auf dieser Grundlage Methoden zur Hydroxyprolin-Bestimmung angegeben (17-20). Rauskolb (20) hat das Verfahren von STEGEMANN, welches Hydroxyprolin durch Oxydation in leicht saurem Milieu nachweist, systematisch auf Störfaktoren untersucht, die von den an der-Reaktion beteiligten Substanzen wirksam werden können. Bei seinen Untersuchungen an Sehnengewebe fand er, daß bei genauer Beachtung der Arbeitsanweisung die Fehlerbreite bei $\pm 5 \%$ liegt. Sie ist vor allem durch die Inhomogenität des Materials bedingt.

In der vorliegenden Arbeit wurden Kälberaortenklappen untersucht, jeweils nativ und nach Einwirkung von fünf verschiedenen Konservierungs- bzw. Sterilisationsverfahren. Die Ergebnisse lassen zwei von den nativen Klappen als Bezugswert deutlich abweichende Gruppen erkennen. Die mit $\beta$-Propiolacton behandelten und die gammastrahlensterilisierten Klappen sind offensichtlich stärker als die Klappensegmente der übrigen Behandlungsgruppen geschädigt worden, da durch die Trypsinwirkung signifikant höhere Hydroxyprolinmengen in den Uberstand abgegeben wurden. Die Lyophilisation allein verursacht offensichtlich keinen zusätzlichen Schaden. Der relativ hohe Hydroxyprolingehalt im Überstand der nativen Klappen läßt allerdings vermuten, $\mathrm{da} B$ die Bearbeitung und Aufbewahrung der Klappensegmente ohne zusätzliche Behandlung doch das Gewebe bereits spürbar schädigt.

Die Sterilisation mit Äthylenoxid und die Cialitbehandlung scheinen das Herzklappengewebe nicht exheblich zu schädigen. Wir vermuten allerdings, daß der signifikant geringere Hydroxyprolinanteil des Überstandes bei der Äthylenoxidbehandlung zu einem Teil auf eine Vernetzung des Kollagens zurückzuführen ist, die die Trypsinwirkung verdeckt. Wir können nicht entscheiden, ob auch bei der Cialitwirkung eine chemische Modifikation die Trypsinwirkung behindert oder verdeckt.

An einer weiteren Untersuchung an menschlicher Dura soll untersucht werden, ob das geschilderte Verfahren allgemein geeignet ist, quantitativ unterschiedliche Schädigungen an kollagenem Fasergewebe anzuzeigen.

\section{Literatur}

1. Ross, D., Brit. J. Surg. 64, 842 (1967). - 2. Barrat-Boyes, B. G., Thorax, London 19, 131 (1964). - 3. BinEt, J. P., A. CARpeNtrer und J. Langlors, Langenbeck's Arch. klin. Chir. 316, 800 (1966). - 4. Ionescu, M. I., Y. S. Masshour und G. H. Wooler, Thorax, London 23, 221 (1968). - 5. Carpentier, A., Traitment des lésions valvulaires aortiques par des greffes hétéroplastiques. R. Foulon et Cie Paris (1966). - 6. Braunwald, N. S. und Don E. Detmer, Progr. Cardiovas. Dis. 11, 113 (1968). 7. Angell, W. W., R. D. Wuerflein und N. E. Shumway, Surgery; S. Louis 62, 807 (1967). - 8. Pra, H. W., Mels. Med. Mitt. 41, 61 (1967). - 9. Weber, K. A. und F. Knipping, Zbl. Chir. 18, 682 (1966). - 10. Weickmann, F. und H. J. Steinke, Chirurgie, Lausanne 30, 320 (1959). - 11. FlosDORF, E. W. und
S. Mudd, J. Immunol., Baltimore 29, 389 (1935). - 12. Colırns, H. A. und J. H. Forster, Amer. Surg. 20, 820 (1954). - 13. MeEKER, I. A. und R. E. Gross, Science (New York) 114, 283 (1951). 14. Szilagyi, D. E., P. R. Overhulse, C. P. Shonnard und G. A. Logrippo, Surg. Forum 5, 244 (1954). - 15. HehrdeIN, F. W., W. Schmitt und N. PapastaVrou, Thoraxchirurgie 17, 244 (1969). 16. RApoport, S. M., Medizinische Biochemie, Verlag Volk und Gesundheit, Berlin (1962). - 17. NeumanN, R. E. und M. A. LoGAN, J. biol. Chemistry 184, 299 (1950). - 18. Prockop, D. W. und S. Udenfriend, Analytic Biochem. 1, 228 (1960). - 19. StEGEMANN, H., Hoppe-Seyler's Zschr. physiol. Chem. 310, 41 (1958). 20. Rauskolb, R., Inaug. Disș. Gießen (1967).

Dr. med. Hans Haas und Prof. Dr. med. L. Róka Institut für Klinische Chemie an den Universitätskliniken 63 Gießen, Klinikstr. 32b

Dr. med. F. W. Hehrlein Chirurgische Universitätsklinik 63 Gießen, Klinikstr. 37 


\section{H. Pilz:}

\section{Die Lipide des normalen und pathologischen Liquor cerebrospinalis}

Mit 4 Abbildungen und 23 Tabellen.

Etwa 135 Seiten. Erscheint April 1970.

(Schriffenreihe Neurologie/Neurology Series, Band 4)

DM 48, -; US $\$ 13.20$

$\bullet$.

Das Buch gibt eine umfassende Übersicht über Untersuchungsmethoden und -ergebnisse von Lipiden des Liquor cerebrospinalis und fügt kritische Betrachtungen über deren Herkunft an.

\section{Arnold: Hisfochemie}

Einführung in Grundlagen und Prinzipien der Methoden

Mit 68 Abbildungen. 218 Seiten. 1968.

Gebunden DM 38,_; US $\$ 10.50$

Eine einprägsame Darstellung der Prinzipien histochemischer Reaktionen. Ein ausgezeichneter Leitfaden für den Anfänger in der Histochemie, aber auch eine wertvolle Sammlung von Methoden. Enthält zahlreiche Hinweise auf die elektronenmikroskopische Histochemie sowie einen Anhang: Verfahrensweisen und Tabellen.

\section{H. N. Christensen:}

\section{Elektrolytstoffwechsel}

Übersetzt von R. und K. Bergmann. Mit 37 Abbildungen. 161 Seiten. 1969.

(Heidelberger Taschenbücher, Band 55) DM 12,80; .US $\$ 3.60$

Kurze und verständliche Darstellung biochemischer und physikalisch-chemischer Grundlagen der Homöostase in den Körperflüssigkeiten. Regulation des Säure-Basen- und des Elektrolythaushalts.

\section{R. E. Dohrmann:}

\section{$\beta$-Glucuronidase}

Mit 26 Abbildungen. 92 Seiten. 1969.

(Experimentelle Medizin, Pathologie und Klinik, Band 28) Gebunden DM 36, -; US $\$ 9.90$

Forschungsbericht über die Eigenschaften der B-Glucuronidase unter besonderer Berücksichtigung der klinisch wichtigen Erkenntnisse. Von diagnostischem Wert z. B. ist die gesteigerte B-Glucuronidase-Aktivität des Serums bei Erkrankungen der Leber und des Pankreas sowie beim Diabetes mellitus und der Arteriosklerose. Außerdem kann die Höhe der B-Glucuronidase-Aktivität als Indikator für die Konzentration der im Organismus vorhandenen Sexualhormone dienen.

\section{R. Gross:}

\section{Medizinische Diagnostik - Grundlagen und Praxis}

Mit 12 Abbildungen und 14 Tabellen. 230 Seiten. 1969. (Heidelberger Taschenbücher, Band 48) DM 9,80; US $\$ 2.70$

Eine allgemeine Methodologie der heutigen Diagnostik mit ihren historischen, ärztlichen, medizinischen, logischen und mathematischen Elementen.

\section{K. Kunze:}

\section{Das Sauerstoffdruckfeld im normalen und pathologisch veränderten Muskel}

Untersuchungen mit einer neuen Methode zur quantitativen Erfassung der Hypoxie in situ.

Mit 67 Abbildungen. 126 Seiten. 1969.

(Schriftenreihe Neurologie/Neurology Series, Band 3)

DM 58, —; US $\$ 16.00$

Die Bedeutung der lokalen Sauerstoffversorgung des Muskels für eine Reihe von neuromuskulären Erkrankungen wird dargelegt. Die benutzte, neve Methodik der Sauerstoffdruckmessung wird ausführlich geschildert.

Bitte Prospekte anfordern! 


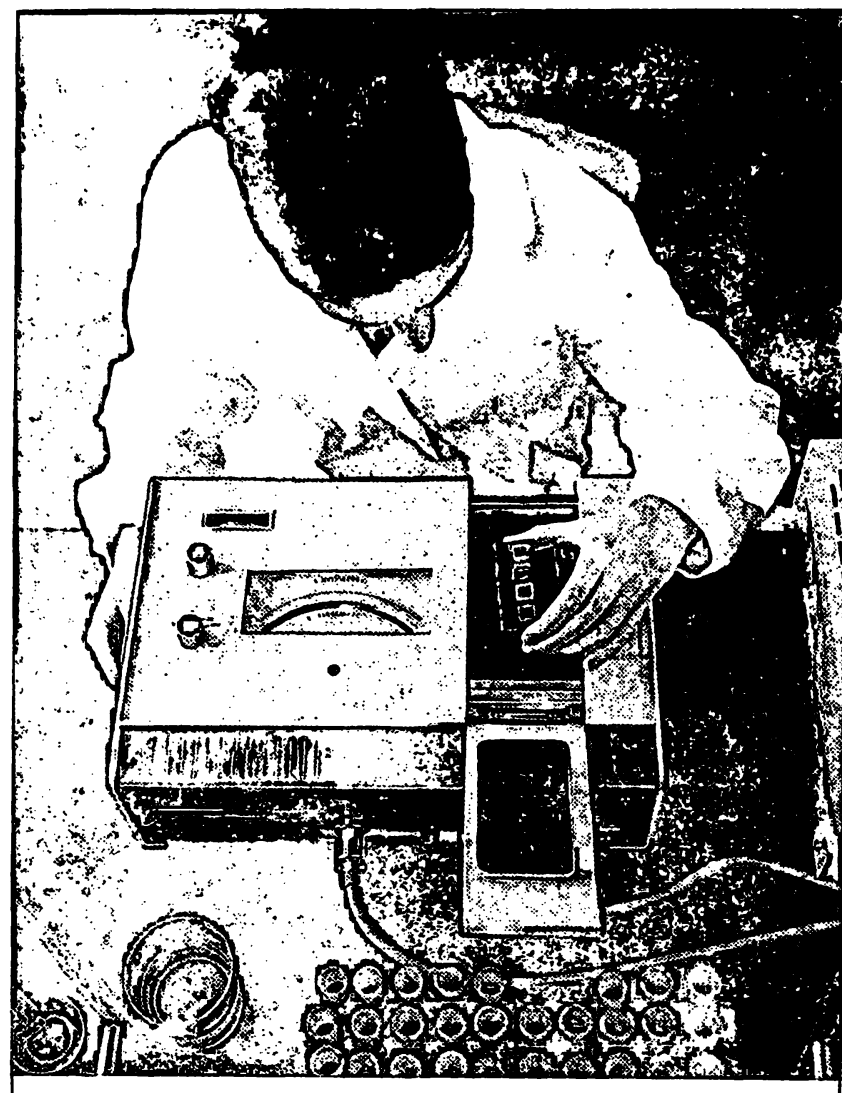

Hitachi-

\section{Spektralphotometer 101}

Hohe Leistung bei günstigem Preis. 3 Modelle für Wellenlängenbereiche von $340-900 \mathrm{~nm}, 220-340 \mathrm{~nm}$ und 220-900 nm.

Optische Ausrüstung mit Gittermonochromator und SpezialPhotozelle für Gesamt-Wellenlängenbereich. Probenraum für 4 Rechteckküvetten, Sonderzubehör für Serienmessungen.

\section{Alleinvertretung für Deutschland:}

\section{Colora Messtechnik GmbH} 7073 Lorch/Württ., Postfach 5 T (07172) 6041, FS 07-248886

Technische Büros (Verkauf und Kundendienst): 1000 Berlin 30, Kurfürstenstraße 84, T 135200 2000 Hamburg 19, Osterstraße 63, T 400606 , FS 02-12947 3000 Hannover, An der Tiefenriede 45, T 884500 4000 Düsseldorf, Kronprinzenstr. 62, T 17860 , FS 08-587 253 6000 Frankfurt a.M., Röderbergweg 4-6, T446031, FS 04-11216 8000 München 2, Dachauer Straße 175, T 5169858$$
\text { BODF }
$$

\section{Aminosäure- Trennungen können Sie mit dieser Apparatur in 15 Minuten ausführen}

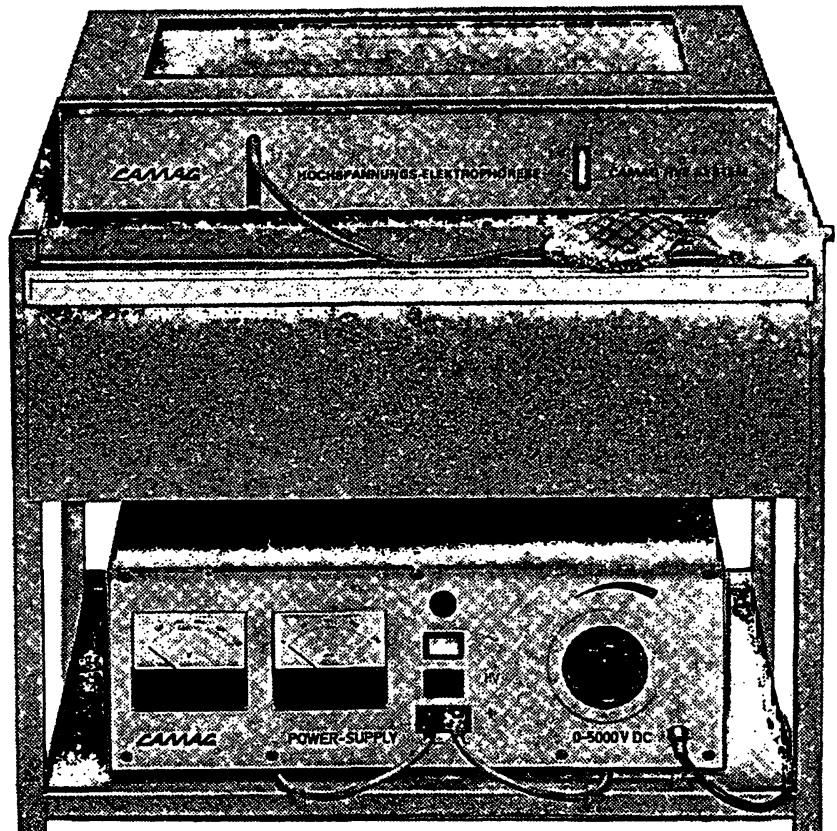

Es ist das neue CAMAG

Hochspannungs-ElektrophoreseSystem mit seinem einzigartigen Kühlprinzip. Wollen Sie wissen, wie das funktioniert? Sie brauchen dazu Leitungswasser und etwas Luft, beides haben Sie ja wohl im Hause. An das Wasser schliessen Sie die Zelle an, und mit der Luft ... aber das zeigen wir Ihnen am besten in Funktion, in Ihrem Hause.

Wenn Sie wollen, schicken wir Ihnen unseren Prospekt, aber lieber kommen wir zu Ihnen mit der ganzen Ausrüstung.

Natürlich können Sie auch andere niedermolekulare Substanzen trennen, z.B.: Indole, Porphyrine, Zucker, Purine, Vitamine, anorganische lonen.

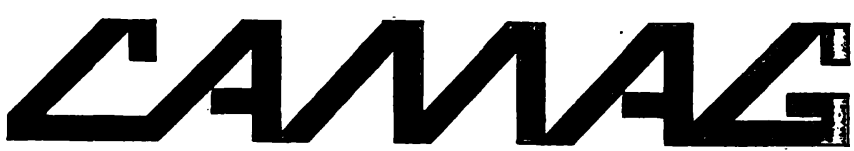

Chemie-Erzeugnisse und Adsorptionstechnik AG Homburgerstrasse 244132 Muttenz/Schweiz

Unser Zweigbetrieb in der Bundesrepublik: CAMAG, 1000 Berlin 45, Baseler Strasse 65 